\title{
Coordinated Multi-Antenna Processing for Cellular Underlay Device-to-Device Communication
}

\author{
Petri Komulainen and Antti Tölli \\ Centre for Wireless Communications, University of Oulu \\ P.O.Box 4500, FI-90014 University of Oulu, Finland \\ email: petri.komulainen@ee.oulu.fi, antti.tolli@ee.oulu.fi
}

\begin{abstract}
We propose linear coordinated transmit-receive beamforming methods for spatial underlay direct device-todevice (D2D) communication in cellular networks where the user terminals (UTs) employ multiple antenna elements. For a D2D terminal pair, direct communication is a beneficial alternative compared to the cellular mode, where the UTs communicate to each other via a base station (BS) that acts as a relay. For mode selection, spatial scheduling, and transmitter-receiver design, we formulate a joint weighted sum-rate (WSR) maximization problem, and adopt an optimization framework where the WSR maximization is carried out via weighted sum mean-squarederror (MSE) minimization. Furthermore, we propose to connect the uplink and downlink design problems - for the case when the BS relays the data - via scalar weight optimization. According to the results, D2D communication can provide very high data rates for the D2D pair and at the same time save resources for other UTs that employ regular cellular connections.
\end{abstract}

\section{INTRODUCTION}

We consider a cellular system model with one multi-antenna base station (BS) and multiple multi-antenna user terminals (UTs). In cellular mode, spatial multiplexing for both uplink (UL) and downlink (DL) user signals is employed so that the DL forms a MIMO broadcast channel (BC) and the UL forms a MIMO multiple access channel (MAC). Furthermore, we assume that the data streams are transmitted via linear spatial precoding (beamforming) and that each receiver treats the signals intended to other receivers as colored noise. The model is further extended by allowing spatial underlay deviceto-device (D2D) communication so that some UT pairs are allowed to directly transmit to each other. As a result, the system model becomes a mixture of MIMO interference channel (IFC), BC, and MAC. Coordinated transmitter and receiver design as well as spatial scheduling is carried out in order to avoid or mitigate inter-link interference.

Compared to the case where the UT pair communicates via the BS, direct D2D connection has multiple benefits [1], [2]. Proximity gain is obtained when the D2D UTs are close to each other so that the channel between them is strong. The gain translates to very low transmit powers or alternatively to high data rates. On the other hand, the duplexing loss and delay caused by multi-hop transmission is avoided, and resources are saved for other cellular users. Thus, from the network perspective, it is beneficial to allow direct D2D communication.

Multi-antenna transmit-receive design for underlay D2D communication by reusing the DL resources was considered already in [3], where the BS transmitter was designed with the aim to avoid creating interference to the D2D receiver. In [4], the BS transmitter and the D2D transmitter were designed with the objective to maximize the sum-rate of the DL slot. In this paper, we formulate the transmitter and receiver design as a joint weighted sum-rate (WSR) maximization problem, where the utility is the aggregate sum of end-to-end data rates carried via UL, DL, and D2D links. Furthermore, we allow the direct D2D link to co-exist with the cellular connection where the D2D terminals communicate via the BS, and optimize these transmission strategies jointly. Since the rates of UL and DL links for the D2D data need to be equal, the UL and the DL transmit-receive problems are coupled. We propose to search for a scalar weight that effectively equalizes these two rates. We show that in certain scenarios, it is beneficial to allow a multi-route connection between the UTs.

When accompanied with just transmit power constraints, the WSR maximization problem is always feasible. The WSR criterion can also be directly linked to the multi-user scheduling problem, and the priority weights of different users may be adaptively adjusted to match their services or to ensure fairness between the users. Furthermore, WSR maximization carries out spatial user and beam scheduling implicitly, and in the context of this paper, it also makes the selection whether the D2D communication is carried out via the BS or via a direct link, or both. However, in the context of interference channels, WSR maximization is not a convex problem with respect to the transmit covariance matrices [5], and, therefore, only local optima can be found via practical methods.

A connection between the weighted sum mean-squarederror (WSMSE) minimization and the WSR maximization problems in the MIMO BC was established in [6]. In this approach, a local WSR optimum is found via alternating optimization of the transmit and receive filters, and by iteratively updating the MSE weights. The same approach was taken in [7] and [8] in order to solve the WSR problem for the MIMO interference channel (IFC) consisting of a set of transmitter and receiver pairs, and [9], [10] generalized the treatment for MIMO interfering BC. Furthermore, [9] formulated a new joint optimization problem of all three variables (transmitters, receivers, weights) that was shown to be equivalent to the WSR maximization problem. One more generalization of the WSMSE method was presented in [11], where the approach was applied to the MIMO X channel where all the transmitters 
are connected to all the receivers, and each transmitter-receiver link conveys a disjoint data stream.

The paper is organized as follows. Section II describes the system model, and Section III presents the system sum-rate maximization problem. Finally, numerical results are given in Section IV, and conclusions are provided in Section V.

\section{System Model And Notation}

In this section, the system model for the cellular system with underlay D2D communication is described. First, a general transmit-receive channel model that can be used for modeling both the UL and the DL slots is given. Then, an example of the UL and DL communication system is drawn as depicted in Fig. 1.

\section{A. General Transmit-Receive Model}

We consider a mixed multi-user MIMO interference system with a set of nodes indexed $k=1,2, \ldots, K$, where the nodes are further split into transmitters and receivers. The system is a mixture of broadcast channel (BC), multiple access channel (MAC), and interference channel (IFC), as each transmitter may transmit to multiple receivers, and each receiver may receive from multiple transmitters. There is a predefined set of candidate communication links indexed $\ell=1,2, \ldots, L$, and each link is associated with one transmitting node and one receiving node. Furthermore, each link carries disjoint data. We denote the index of the transmitting node related to link $\ell$ as $t_{\ell}$, and the index of the corresponding receiving node as $k_{\ell}$. All the links are spatially multiplexed into the same time-frequency slot, and we assume that the whole network is symbol-synchronous.

Let the MIMO channel matrix between a transmitting node $t$ and a receiving node $k$ be $\mathbf{H}_{k}^{t} \in \mathbb{C}^{N_{k} \times N_{t}}$, where $N_{k}$ and $N_{t}$ are the number of antenna elements employed by node $k$ and $t$, respectively. The complex downlink MIMO signal received by any node $k$ can be expressed as

$$
\mathbf{x}_{k}=\sum_{\ell} \mathbf{H}_{k}^{t_{\ell}} \mathbf{B}_{\ell} \mathbf{s}_{\ell}+\mathbf{z}_{k}
$$

where the transmit precoder matrix for link $\ell, \mathbf{B}_{\ell}$, has maximum dimensions $N_{t_{\ell}} \times \min \left(N_{k_{\ell}}, N_{t_{\ell}}\right)$. Similarly, the data symbols are gathered into the vector $\mathbf{s}_{\ell}$. Here, the data streams are independent and have zero mean and unit power so that $\mathbb{E}\left[\mathbf{s}_{\ell} \mathbf{s}_{\ell}^{\mathrm{H}}\right]=\mathbf{I}$. Furthermore, $\mathbf{z}_{k} \in \mathbb{C}^{N_{k}}$ is a complex white Gaussian noise vector with variance $\mathcal{N}_{0}$ per element. Note that in the model, the number of data streams transmitted by node $t$ may be larger than $N_{t}$. However, as a result of the precoder optimization, the transmit powers or the norms of the precoding vectors of many data streams become zero so that the system will not be spatially overloaded.

For link $\ell$, define the mean-squared-error (MSE) matrix as

$$
\mathbf{E}_{\ell} \triangleq \mathbb{E}\left[\left(\mathbf{s}_{\ell}-\mathbf{A}_{\ell} \mathbf{x}_{k_{\ell}}\right)\left(\mathbf{s}_{\ell}-\mathbf{A}_{\ell} \mathbf{x}_{k_{\ell}}\right)^{\mathrm{H}}\right]
$$

where $\mathbf{A}_{\ell}$ is a linear receiver matrix employed by node $k_{\ell}$. When employing the MMSE receiver, the MSE matrix

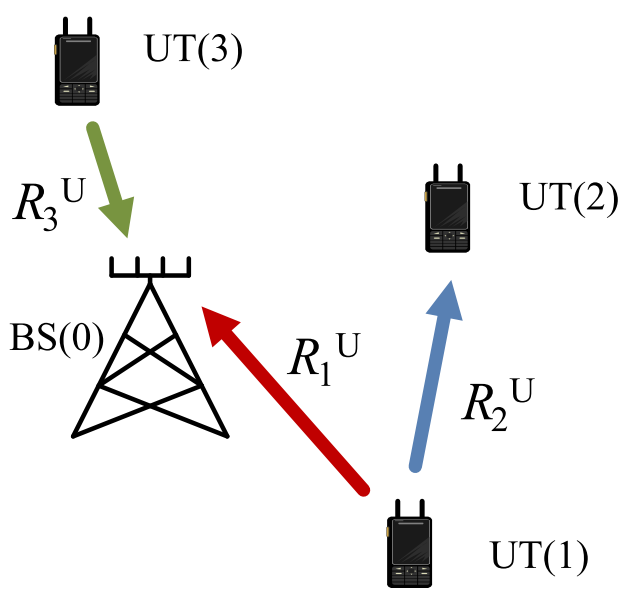

(a)

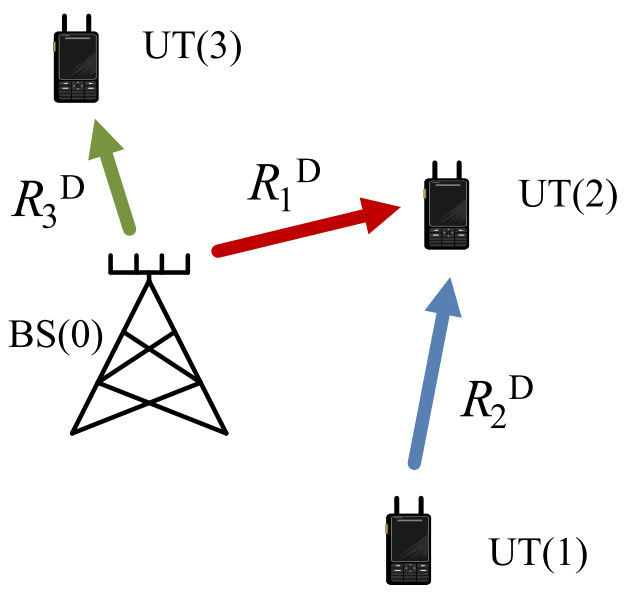

(b)

Fig. 1. (a) Uplink slot. (b) Downlink slot.

becomes [12, Theorem 12.1]

$$
\begin{aligned}
\tilde{\mathbf{E}}_{\ell} & =\mathbf{I}-\left(\mathbf{H}_{k_{\ell}}^{t_{\ell}} \mathbf{B}_{\ell}\right)^{\mathrm{H}} \mathbf{R}_{k_{\ell}}^{-1} \mathbf{H}_{k_{\ell}}^{t_{\ell}} \mathbf{B}_{\ell} \\
& =\mathbf{I}-\mathbf{A}_{\ell}^{\mathrm{MMSE}} \mathbf{H}_{k_{\ell}}^{t_{\ell}} \mathbf{B}_{\ell}
\end{aligned}
$$

where $\mathbf{R}_{k_{\ell}}$ is the received signal covariance matrix at node $k_{\ell}$. For any receiving node $k$, the covariance is

$$
\mathbf{R}_{k} \triangleq \mathbb{E}\left[\mathbf{x}_{k} \mathbf{x}_{k}^{\mathrm{H}}\right]=\sum_{\ell} \mathbf{H}_{k}^{t_{\ell}} \mathbf{B}_{\ell}\left(\mathbf{H}_{k}^{t_{\ell}} \mathbf{B}_{\ell}\right)^{\mathrm{H}}+\mathcal{N}_{0} \mathbf{I}
$$

The achievable rate for link $\ell$, assuming that signals of other links are treated as colored noise, becomes [9]

$$
R_{\ell}=\log \operatorname{det}\left(\tilde{\mathbf{E}}_{\ell}^{-1}\right) \text {. }
$$

Finally, the weighted sum rate (WSR) objective to be maximized can be written as

$$
\sum_{\ell} u_{\ell} R_{\ell}
$$

where $u_{\ell}$ is some non-negative priority weight for link $\ell$. In this paper, we adopt the well-known optimization framework where the WSR maximization is carried out via weighted sum 
MSE minimization [6], [9]. The objective to be minimized can be written as [9]

$$
\sum_{\ell} u_{\ell}\left(\operatorname{Tr}\left(\mathbf{W}_{\ell} \mathbf{E}_{\ell}\right)-\log \operatorname{det}\left(\mathbf{W}_{\ell}\right)\right)
$$

where $\mathbf{W}_{\ell}$ is a positive semidefinite MSE weight matrix for link $\ell$. Here, objective (7) is iteratively minimized with respect to transmit precoders $\mathbf{B}_{\ell}$, receive filters $\mathbf{A}_{\ell}$, and MSE weights $\mathbf{W}_{\ell}$ to find a locally optimal point.

\section{B. Uplink and Downlink with D2D}

In cellular systems, the radio resources dedicated to UL and DL are traditionally separated so that either different frequencies (FDD) or time slots (TDD) are used. The UL and DL slots form two different transmitter and receiver design problems.

Fig. 1(a) depicts an example of the uplink, and Fig. 1(b) shows a related downlink communication system. We denote the transmit precoders in the UL slot as $\mathbf{B}_{\ell}^{\mathrm{U}}, \ell=1,2,3$, and the transmit precoders in the DL slot as $\mathbf{B}_{\ell}^{\mathrm{D}}, \ell=1,2,3$. Similarly, the achievable data rates given by (5) are denoted $R_{\ell}^{\mathrm{U}}$ and $R_{\ell}^{\mathrm{D}}, \ell=1,2,3$, for the UL and DL, correspondingly. Furthermore, the nodes are indexed so that the BS is node 0, and UTs are the nodes $1,2,3$.

In this model, UT(1) wishes to transmit data to UT(2). There are two choices: the data can be relayed via the BS as in regular cellular access, or direct transmission can be used. When the BS employs decode-and-forward relaying, the rate achievable via the BS becomes

$$
\min \left(R_{1}^{\mathrm{U}}, R_{1}^{\mathrm{D}}\right)=\frac{1}{2}\left(R_{1}^{\mathrm{U}}+R_{1}^{\mathrm{D}}\right),
$$

if the precoders were properly designed so that UL and DL rates are equal. On the other hand, assuming that D2D transmission is allowed both during the UL and the DL slots, the achievable direct transmission rate becomes $R_{2}^{\mathrm{U}}+R_{2}^{\mathrm{D}}$. If joint cellular and D2D access is allowed, separate data streams can be transmitted via the two alternative routes.

The system model also contains a third UT that is engaged in regular UL-DL cellular communication, and it shares the spatial resources with the other two UTs. In order to quantify its cellular access rate with a single utility that is comparable to the cellular access rate between UT(1) and UT(2), we define this utility similarly to (8) as $\frac{1}{2}\left(R_{3}^{\mathrm{U}}+R_{3}^{\mathrm{D}}\right)$.

\section{TRANSMITTER OPTIMIZATION}

Denote a composite variable containing all the precoders of the $\mathrm{UL}$ slot as $\mathbf{B}^{\mathrm{U}}=\left(\mathbf{B}_{1}^{\mathrm{U}}, \mathbf{B}_{2}^{\mathrm{U}}, \mathbf{B}_{3}^{\mathrm{U}}\right)$, and similarly a composite variable containing all the precoders of the DL slot as $\mathbf{B}^{\mathrm{D}}=$ $\left(\mathbf{B}_{1}^{\mathrm{D}}, \mathbf{B}_{2}^{\mathrm{D}}, \mathbf{B}_{3}^{\mathrm{D}}\right)$. Furthermore, let $\mathbf{B}=\left(\mathbf{B}^{\mathrm{U}}, \mathbf{B}^{\mathrm{D}}\right)$ contain all the UL and DL precoders, and define the system end-to-end sum rate as

$$
f(\mathbf{B}) \triangleq \min \left(R_{1}^{\mathrm{U}}, R_{1}^{\mathrm{D}}\right)+R_{2}^{\mathrm{U}}+R_{2}^{\mathrm{D}}+\frac{1}{2}\left(R_{3}^{\mathrm{U}}+R_{3}^{\mathrm{D}}\right) .
$$

We wish to find the transmit precoders that maximize $f$, i.e., to solve the problem

$$
\begin{aligned}
\operatorname{maximize} & f(\mathbf{B}) \\
\text { s.t. } & \sum_{\ell \in \mathcal{L}_{t}^{U}} \operatorname{Tr}\left(\mathbf{B}_{\ell}^{\mathrm{U}}\left(\mathbf{B}_{\ell}^{\mathrm{U}}\right)^{\mathrm{H}}\right) \leq P_{t}, \quad t=1,3 \\
& \sum_{\ell \in \mathcal{L}_{t}^{D}} \operatorname{Tr}\left(\mathbf{B}_{\ell}^{\mathrm{D}}\left(\mathbf{B}_{\ell}^{\mathrm{D}}\right)^{\mathrm{H}}\right) \leq P_{t}, \quad t=0,1,
\end{aligned}
$$

where $P_{t}$ is the maximum transmit power constraint for node $t$. Furthermore, $\mathcal{L}_{t}^{U}$ and $\mathcal{L}_{t}^{D}$ denote the set of links for which node $t$ is the transmitter. Here, $\mathcal{L}_{1}^{U}=\{1,2\}, \mathcal{L}_{3}^{U}=\{3\}$, $\mathcal{L}_{0}^{D}=\{1,3\}$, and $\mathcal{L}_{1}^{D}=\{2\}$.

Let us define an upper bound of $f$,

$$
g(\mathbf{B}, \alpha) \triangleq \alpha R_{1}^{\mathrm{U}}+(1-\alpha) R_{1}^{\mathrm{D}}+R_{2}^{\mathrm{U}}+R_{2}^{\mathrm{D}}+\frac{1}{2}\left(R_{3}^{\mathrm{U}}+R_{3}^{\mathrm{D}}\right)
$$

for which it holds that $f(\mathbf{B}) \leq g(\mathbf{B}, \alpha)$ for any $0 \leq \alpha \leq 1$. Thus, if for some $\alpha$ we are able to find transmit precoders $\mathbf{B}^{\star}$ that maximize $g$, and it turns out that $f\left(\mathbf{B}^{\star}\right)=g\left(\mathbf{B}^{\star}, \alpha\right)$, we have also found the optimal solution of the original problem (10).

For fixed $\alpha$, maximizing of $g$ decouples into two separate maximization problems, one for the UL slot and another for the DL slot. The corresponding objectives are

$$
\begin{aligned}
& g_{\mathrm{U}}\left(\mathbf{B}^{\mathrm{U}}, \alpha\right) \triangleq \alpha R_{1}^{\mathrm{U}}+R_{2}^{\mathrm{U}}+\frac{1}{2} R_{3}^{\mathrm{U}} \\
& g_{\mathrm{D}}\left(\mathbf{B}^{\mathrm{D}}, \alpha\right) \triangleq(1-\alpha) R_{1}^{\mathrm{D}}+R_{2}^{\mathrm{D}}+\frac{1}{2} R_{3}^{\mathrm{D}},
\end{aligned}
$$

and the UL optimization problem is to maximize (14) subject to constraint (11), and the DL optimization problem is to maximize (15) subject to constraint (12). Now, the two objectives have the WSR form as in (6). Thus, we seek for the maximum of (14) and (15) by casting the problems into form (7), and by applying the well-known method of WSR maximization via weighted sum MSE minimization [6], [9]. In this scheme, transmit precoders, receive filters, and MSE weights are iteratively optimized.

Denote the optimal values of $R_{1}^{\mathrm{U}}$ and $R_{1}^{\mathrm{D}}$ for a given $\alpha$ as $R_{1}^{\mathrm{U} \star}(\alpha)$ and $R_{1}^{\mathrm{D} \star}(\alpha)$, respectively. A sufficient condition for the equality $f\left(\mathbf{B}^{\star}\right)=g\left(\mathbf{B}^{\star}, \alpha\right)$, and thus for the optimality with respect to problem (10), is that $R_{1}^{\mathrm{U} \star}(\alpha)=R_{1}^{\mathrm{D} \star}(\alpha)$. This is logical since if the rates $R_{1}^{\mathrm{U}}$ and $R_{1}^{\mathrm{D}}$ are unequal, it means that one of the two links has been assigned with more power or spatial resources than can be utilized. These excess resources could be assigned to benefit $R_{2}^{\mathrm{U}}$ or $R_{3}^{\mathrm{D}}$ instead, to further increase the objective (9).

It can be seen from objective (14) that $R_{1}^{\mathrm{U}}(\alpha)$ is monotonically increasing in $\alpha$. Similarly, it can be seen from (15) that $R_{1}^{\mathrm{D}}(\alpha)$ is monotonically decreasing in $\alpha$. Thus, we may search for $\alpha$ via bi-section in the range $(0,1)$ until the equality is found. This way, the maximization of (14) and (15) is carried out multiple times. Note that a point of equality may not always exist since the optimal rates might be discontinuous in $\alpha$. The procedure is summarized in Algorithm 1 .

Finally, it is worth noting that objectives (14) and (15) constitute non-concave maximization problems, for which only 


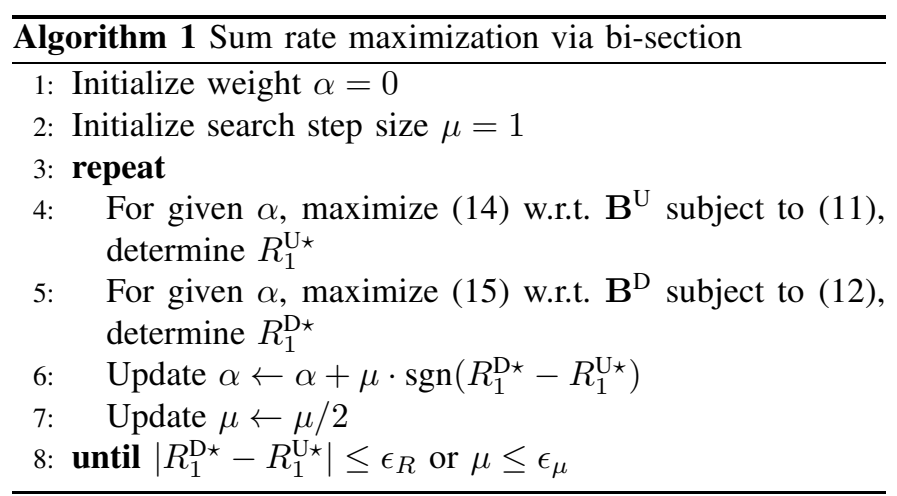

locally optimal solutions can be found via practical means. Thus, in practice, we cannot guarantee to find the global maximum of $g$ or $f$ either.

\section{NUMERICAL RESULTS}

The network of Fig. 1 was simulated in quasistatic frequency flat Rayleigh fading with uncorrelated channels between antennas. All the UTs employ two antennas each, and the BS employs four antenna elements. The average channel gain between all three UTs and the BS was normalized to 0dB. It was also assumed that UT(3) is on the other side of the BS compared to the other two UTs so that the average channel gain between them is $-9 \mathrm{~dB}$. The average channel gain between UT(1) and UT(2) was varied as a parameter. Transmit power constraints were set equal for all transmitting nodes $t=0,1,3$ so that $P_{t} / \mathcal{N}_{0}=5 \mathrm{~dB}$.

Three different options for the connection between UT(1) and UT(2) were simulated: joint cellular and D2D communication, direct D2D communication only, and cellular access only. Furthermore, two options for the direct D2D link were tested: the case were only the UL slot is used for the direct link, and the case were both UL and DL slots are used.

The results are shown in Fig. 2(a) in terms of the system sum rate utility (9) as a function of average direct D2D channel gain. As can be seen, the joint design always outperforms the other designs. However, when the D2D channel becomes stronger, the rate provided by direct D2D transmissions increases rapidly, and the additional gain from the joint design disappears.

Fig. 2(b) shows the sum rate of the UT(1)-UT(2) end-toend connection. As can be seen, the gain from increasing D2D channel gain is dramatic, and allowing the use of both UL and DL slots approximately doubles the end-to-end rate. Finally, Fig. 2(c) depicts what happens to the rate of UT(3). Interestingly, allowing direct D2D connection for the other two UTs mostly increases the rates of UT(3) as well. This is due to the fact that the direct D2D reserves less of cellular resources that can then be used by UT(3).

\section{CONCLUSION}

We have proposed linear coordinated transmit-receive beamforming methods for spatial underlay direct D2D communication in cellular networks where the UTs employ multiple

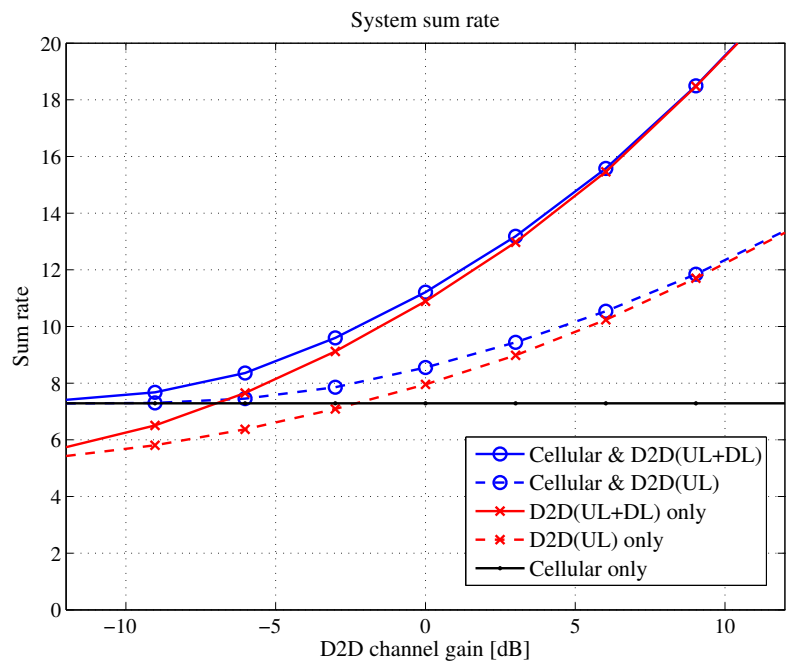

(a)

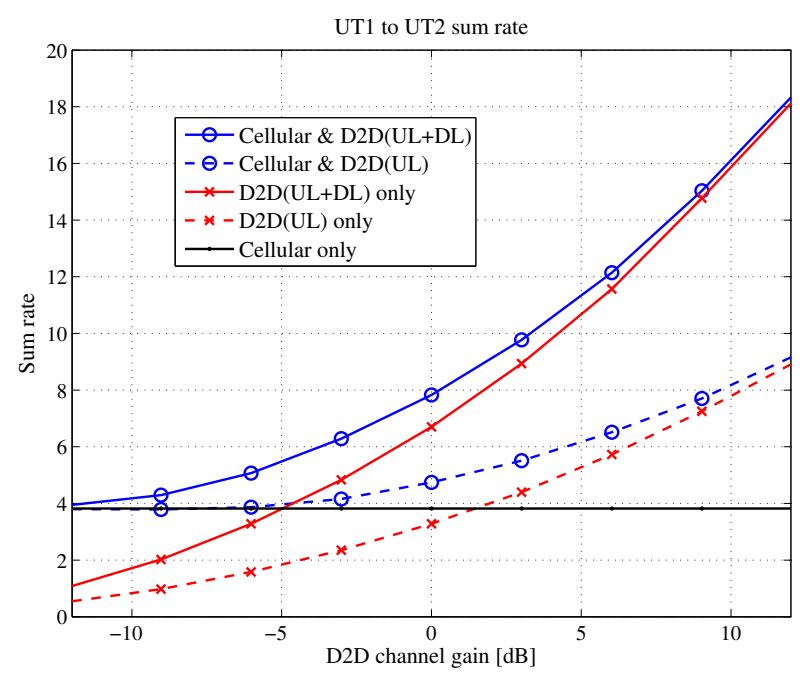

(b)

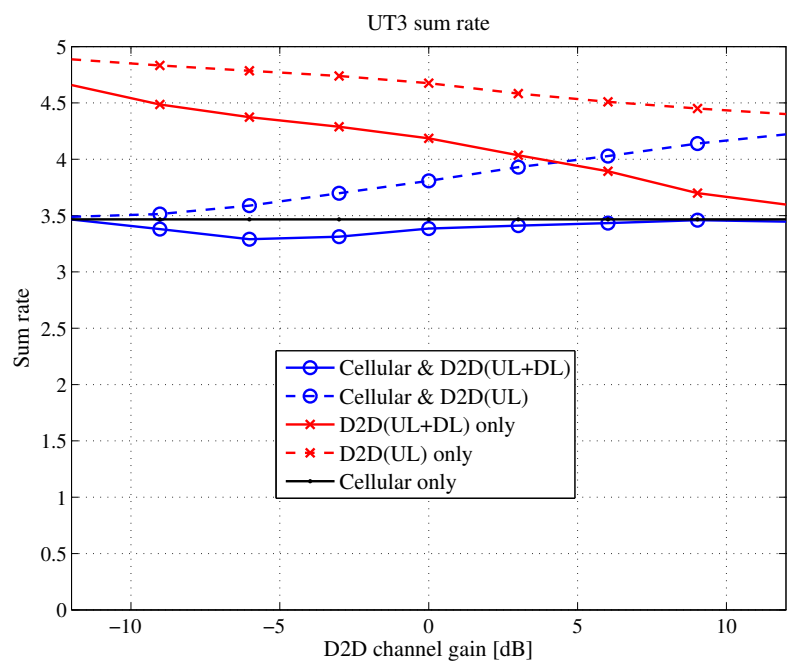

(c)

Fig. 2. (a) System sum rate. (b) End-to-end rate between UT1 and UT2. (c) Sum rate for UTO. 
antenna elements. We formulated the transmitter and receiver design as a WSR maximization problem, where the utility is the aggregate sum of end-to-end data rates carried via UL, DL, and D2D links. The direct D2D link was allowed to coexist with the cellular connection where the D2D terminals communicate via the $\mathrm{BS}$, and these transmission strategies were optimized jointly. We showed that in certain scenarios, it is beneficial to allow a multi-route connection between the UTs. For a D2D terminal pair, direct communication is almost always a beneficial alternative compared to regular cellular access.

The proposed optimization algorithm was presented in a centralized form that can be carried out by the BS based on global channel knowledge. The BS also needs to signal the optimized precoders to the corresponding UTs. To this end, the BS needs to first obtain information of the channel realizations between all transmitting and receiving nodes. Compared to the cellular access, the additional signaling load caused by allowing the underlay D2D communication is formed by the need to estimate and report the cross-channels between all the UTs to the BS. The signaling load remains reasonable if the group of spatially multiplexed terminals is kept relatively small.

\section{ACKNOWLEDGMENT}

This work has been supported by the Finnish Funding Agency for Technology and Innovation (Tekes), Nokia, Huawei Finland, and Broadcom Communications Finland.

\section{REFERENCES}

[1] G. Fodor, E. Dahlman, G. Mildh, S. Parkvall, N. Reider, G. Miklos, and Z. Turanyi, "Design aspects of network assisted device-to-device communications," IEEE Commun. Mag., pp. 170-177, Mar. 2012.

[2] D. Feng, L. Lu, Y. Yuan-Wu, G. Y. Li, G. Feng, and S. Li, "Deviceto-device communications underlaying cellular networks," IEEE Trans. Commun., vol. 61, no. 8, pp. 3541-3551, Aug. 2013.

[3] P. Jänis, V. Koivunen, C. B. Ribeiro, K. Doppler, and K. Hugl, "Interference-avoiding MIMO schemes for device-to-device radio underlaying cellular networks," in Proc. IEEE Int. Symp. Pers., Indoor, Mobile Radio Commun., Tokyo, Japan, Sep. 13-16 2009, pp. 2385-2389.

[4] D. Zhu, W. Xu, H. Zhang, C. Zhao, J. C. F. Li, and M. Lei, "Ratemaximized transceiver optimization for multi-antenna device-to-device communications," in Proc. IEEE Wireless Commun. and Networking Conf., Shanghai, China, Apr.7 -10 2013, pp. 4152-4157.

[5] S. Ye and R. S. Blum, "Optimized signaling for MIMO interference systems with feedback," IEEE Trans. Signal Processing, vol. 51, no. 11, pp. 2839-2848, Nov. 2003.

[6] S. S. Christensen, R. Agarwal, E. Carvalho, and J. M. Cioffi, "Weighted sum-rate maximization using weighted MMSE for MIMO-BC beamforming design," IEEE Trans. Wireless Commun., vol. 7, no. 12, pp. 4792-4799, Dec. 2008.

[7] D. A. Schmidt, C. Shi, R. A. Berry, M. L. Honig, and W. Utschick, "Minimum mean squared error interference alignment," in Proc. Annual Asilomar Conf. Signals, Syst., Comp., Nov. 2009, pp. 1106-1110.

[8] F. Negro, S. P. Shenoy, I. Ghauri, and D. T. M. Slock, "Weighted sum rate maximization in the MIMO interference channel," in Proc. IEEE Int. Symp. Pers., Indoor, Mobile Radio Commun., Istanbul, Turkey, Sep. 2010, pp. 684-689.

[9] Q. Shi, M. Razaviyayn, Z.-Q. Luo, and C. He, "An iteratively weighted MMSE approach to distributed sum-utility maximization for a MIMO interfering broadcast channel," IEEE Trans. Signal Processing, vol. 59, no. 9, pp. 4331-4340, Sep. 2011.
[10] P. Komulainen, A. Tölli, and M. Juntti, "Decentralized beam coordination via sum rate maximization in TDD multi-cell MIMO systems," in Proc. IEEE Int. Symp. Pers., Indoor, Mobile Radio Commun., Toronto, Canada, Sep. 11 - 14 2011, pp. 1346-1350.

[11] A. Agustin and J. Vidal, "Weighted sum rate maximization for the MIMO X channel through MMSE precoding," in Proc. IEEE Int. Symp. Inform. Theory, Jun. 31 - Aug. 5 2011, pp. 2572-2576.

[12] S. M. Kay, Fundamentals of Statistical Signal Processing: Estimation Theory. Englewood Cliffs, NJ, USA: Prentice-Hall, 1993. 